\title{
FPGA based Image Enhancement using KNN and PSO with BM3D
}

\author{
Mandar D. Sontakke, Meghana Kulkarni
}

\begin{abstract}
Picture upgrade utilizing streamlined strategies alongside advanced channels is need of current period. A tale system is proposed right now, have KNN based pixels orders technique. This arrangement is utilized to distinguish commotion level and just boisterous pixels are prepared utilizing upgraded BM3D utilizing molecule swarm improvement. The outside gathering pixels are brought once again into bunch in this way expelling the clamor. The procedure is additionally trailed by goals improvement and Retinex for dynamic introduction reason. The FPGA usage is done to approve the aftereffects of upgrade. The performance evaluation of the proposed method shows better results over other techniques using FPGA experimentation.

Index Terms: PSO, KNN, BM3D, nonlinear noise delineation, Retinex, FPGA, Resolution enhancement.
\end{abstract}

\section{INTRODUCTION}

Noise in the image may get added at the time of capturing and at the time of transmission. Commotions are having attributes as for their classes. Mainly linear and non linear noise are the main types of noises that get get introduced in the image therby degrading the appearance. Hence based on these types methods are developed for delineation of noises. The evacuation of commotion relies upon the preparing capacities for specific clamor type if there should arise an occurrence of straight clamors. If there should be an occurrence of non-straight commotion types, mix of techniques can be utilized to get the ideal outcomes. The results of noise removal depends on the density of the noise with respect to actual image size that defines total number of pixels in that image the measure of which is performed using mean square error estimation with respect to noiseless image. The area occupied by the noise pixels is the primary concern while evaluating the density of the noise in specific image and noise removal processing. The clamor model has limits while identifying with specific sort and shows loads of constraints when utilized for non-direct commotion evacuation channel structure [1-3]. Assortment of strategies can be seen so far which can be utilized to expel commotion from the picture and show that outcomes rely upon clamor models considered while structuring the clamor evacuation channels. The warm impact and its impact of clamor age is fundamental driver of

Revised Manuscript Received on April 02, 2020.

* Correspondence Author

Mr. Mandar D. Sontakke *, Department of PG Studies, Visvesaraya Technological University, Belgaum, Karnataka, India.

Dr. Meghana Kulkarni, Department of PG Studies, Visvesaraya Technological University, Belgaum, Karnataka, India.

(C) The Authors. Published by Blue Eyes Intelligence Engineering and Sciences Publication (BEIESP). This is an open access article under the CC BY-NC-ND license (http://creativecommons.org/licenses/by-nc-nd/4.0/) nonlinear commotion. The clamor evacuation process includes stages, for example, location and expulsion. While distinguishing the non-direct commotion, existing models show constrained methodology and henceforth versatile strategies are utilized to recognize and expel them. The principle worry at the hour of commotion evacuation is to hold a lot of unique data and less misfortunes. These necessities are opposing to one another and are hard to accomplish. It can b observed that the variety of methods [4-6] are showing good results of nosie removal even in high noise levels. The main challange is to identify the noise pixels in case of non linear noise and hence there is need of preprocessing as mentioned in this paper to distinguish the noise pixels and regular pixels.

\section{RELATED WORK}

Various researchers have developed variety of methods for non-linear noise removal. Hardly any papers are tended to here by considering the procedures utilized and their execution practicality as devoted framework and execution of the framework.

In paper [7], submerged picture improvement is indicated which utilizes combination based methodology. A solitary dim picture based this strategy utilizes dehazing and differentiate upgrades which gives amendment in hued appearances of the information picture. The perceivability is improved alongside expulsion of bends. The weight map method is used in which objects are classified using weight maps in the image. The information sources and loads are mixed utilizing multi-scale combination method. The last picture acquired contains most huge highlights of the picture accordingly keeping their misfortunes immaterial. Submerged murky pictures are conceivable to improve as far as quality with the basic methodology in viable way.

In paper [8], transmittance adjustment strategy is utilized to upgrade the submerged pictures by disregarding the polarization impacts. The basic calculation is fit for upgrading the pictures by changing the luminosity of the pixels with non negative adjustements. The process is repeated untill all noise pixels are delineated from the image thereby resulting in improved image. The technique in paper shows better execution contrasted with past strategies.

In paper [9], underwater image mprovement is shown by removal of noise. The light distribution factor in image in terms of dimness and brightness is considered for classifying the pixels as noise pixels in thich light attenuated by the shallow water is main cause of spreading of of light.

Published By:

Blue Eyes Intelligence Engineering

\& Sciences Publication

(C) Copyriaht: All riahts reserved.

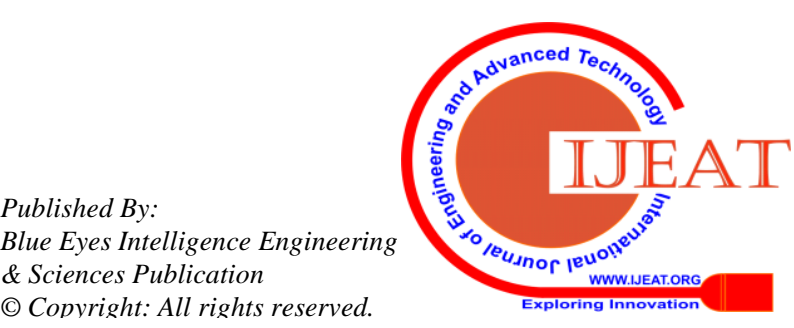


The experimental setup consist of autonomous vehicles sent underwater for the performance evaluation of capturing process.

The ground-breaking object recognition framework right now accomplished with assistance of improved symbolism process which shows accuracy in results while isolating flotsam and jetsam from different articles.

In paper [10], the submerged cloudy picture upgrade is appeared with the utilization of wavelet based methodology. The low differentiation and shading modification issues are tended to. The outcomes got through this strategy gives promising outcomes.

In paper [11], a framework is indicated which gives open source stage to consecutive picture handling. The handling may include the utilization of item discovery and arrangement alongside stereo alignment. The writing computer programs is basic and gives multithreading bolster in this way speeding up. A similar undertaking for various pictures at the same time are conceivable by utilizing this stage for the relevant experimentations.

In paper [12], a strategy is demonstrated which comprises of two phases. At the primary stage, the difference rectification procedure is applied to the picture, where the picture is processed with the adjusted Von Kries theory and extending the picture into two diverse force pictures at the normal incentive as for Rayleigh appropriation. At the subsequent stage, the shading rectification procedure is applied to the picture where the picture is first changed over into tone immersion esteem (HSV) shading model. The change of the shading segment builds the picture shading execution. Subjective and quantitative examinations show that the proposed technique outflanks other cutting edge strategies as far as complexity, subtleties, and clamor decrease.

In paper [13], clamor decrease techniques are talked about which utilize dark worth substitution. Likewise wavelet change based outcomes are acquired which show acceptable execution. Mean square blunder parameter is utilized alongside PSNR for assessing the presentation of the framework.

In paper [14], submerged picture upgrade is done in two stages. In the initial step Haze in the submerged picture is expelled utilizing dull channel earlier. In the subsequent advance Hue modifications dealt with by wavelength remuneration. When luminosity distribution map is determined, luminance with respect to distance and original level inside the picture can be isolated and looked at. The hue of the picture is adjusted with the help of RGB channel values in the image. In the wake of figuring the scale estimation of each RGB part, wavelength is repaid together with the normal RGB and scale estimation of each direct in the picture. Execution of the technique for wavelength remunerated picture is assessed utilizing the PSNR, Focus Measurement, Contrast Improvement Index, and Feature Similarity Index. Execution estimation of the wavelength pay creates preferred improvement results over existing strategy.

In paper [15], differentiate constrained versatile histogram evening out strategy is utilized for upgrading the submerged pictures. The dull channel estimation dependent on lessening of counterfeit light utilized during picture catching procedure is finished. The visual quality is upgraded during experimentation.
In paper [16] preprocessing methods of submerged pictures are engaged. Submerged picture pre-handling is totally important because of the nature of pictures caught submerged. An endeavor has been made to think about and assess the exhibition of three popular channels to be specific, homomorphic channel, anisotropic dispersion and wavelet denoising by normal channel utilized for submerged picture pre-preparing. Out of the three channels, wavelet denoising by normal channel gives alluring outcomes as far as Peak Signal to Noise Ratio and Mean Square Error. Anyway the slipped by time of the three channels is likewise concentrated to recognize the reasonable channels that procedure the picture rapidly by protecting the picture quality.

In paper [17], a double band submerged picture denoising and upgrade calculation, first the first picture was decayed into high-recurrence part $\mathrm{H}$ and low recurrence part $\mathrm{L}$, and afterward $\mathrm{H}$ was sifted into $\mathrm{F}$ by mean move calculation which was improved by utilizing the middle of the road cycle results. A complexity improvement strategy was proposed dependent on the murkiness imaging model and was applied on $\mathrm{L}$ and $\mathrm{F}$. try results exhibit the viability of the proposed calculation.

In paper [18] the strategy for submerged picture upgrade is indicated dependent on the measure of constriction comparing to every wavelength, shading change pay is led and shading balance is reestablished. Impact of clamor is likewise diminished by utilizing the spatial channel. Utilizing this system the perceivability and shade of the picture can be improved.

In paper [19], WCID calculation for submerged picture upgrade is appeared. WCID implies wavelength remuneration and picture dehazing. We additionally think about different submerged pictures preparing strategies result with WCID calculation.

In paper [20] picture division of submerged pictures utilizing molecule swarm streamlining alongside fluffy c implies bunching approach is appeared. The proficient and rapid methodology is seen which utilizes the weighted histogram along these lines changing the enrollment of fluffy based bunches. Likewise, nature of submerged pictures supposedly is improved utilizing the methodology. The division nature of the strategy is improved when seen with results on different pictures. The technique shows upgrade in effective calculation and henceforth, less multifaceted nature.

\section{PROPOSED WORK}

The clustering method is used to classify the pixels in 9 different classes for which KNN classifier method is used. The 8 bit value which lies within 0-255 range is divided in 9 ranges as shown in table 1 .

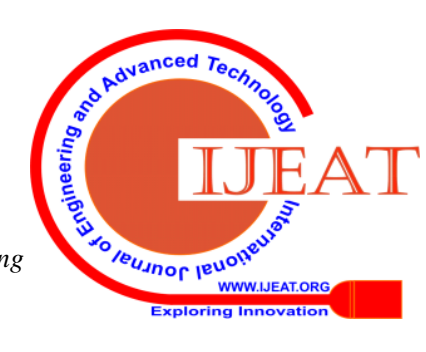




\subsection{Pixel Intensity mapping:}

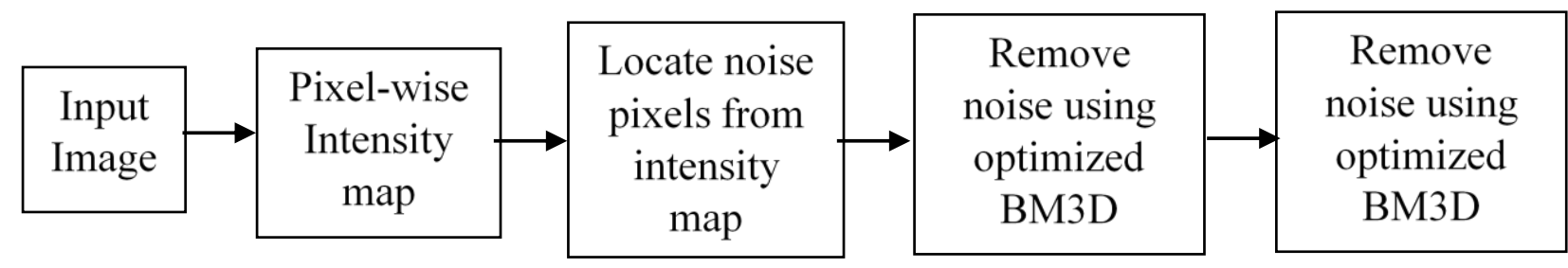

Figure1: System block diagram

into consideration. The dark estimation of these pixels is determined. On the off chance that every pixel has various qualities, at that point clamor should be thick. The classification table is used to identify each pixel with respect to its class name. The window of 9 pixels from which each pixel is classified and identified with its class name is as shown in figure 2 (a) and (b). The pixels having different class is the noise pixel which can easily be identified from the set of 9 pixels as shown in figure 2 (b) which pixel P13. In similar fashoin when window of 3x3 pixels slides for next region, same pixel is again highlighted and hence shows consistancy in the identification strategy.

Table I: Grey values of pixels and respective class name

\begin{tabular}{|c|c|}
\hline $\begin{array}{c}\text { Grey } \\
\text { value } \\
\text { range }\end{array}$ & Class \\
\hline $0-28$ & 1 \\
\hline $29-56$ & 2 \\
\hline $57-84$ & 3 \\
\hline $85-112$ & 4 \\
\hline $113-140$ & 5 \\
\hline $141-168$ & 6 \\
\hline $169-196$ & 7 \\
\hline $197-224$ & 8 \\
\hline $225-255$ & 9 \\
\hline
\end{tabular}

\begin{tabular}{|l|l|l|}
\hline $\mathrm{P}_{11}$ & $\mathrm{P}_{12}$ & $\mathrm{P}_{13}$ \\
\hline $\mathrm{P}_{21}$ & $\mathrm{P}_{22}$ & $\mathrm{P}_{23}$ \\
\hline $\mathrm{P}_{31}$ & $\mathrm{P}_{32}$ & $\mathrm{P}_{33}$ \\
\hline
\end{tabular}

(a)

\begin{tabular}{|l|l|l|}
\hline 4 & 4 & 8 \\
\hline 4 & 4 & 4 \\
\hline 4 & 4 & 4 \\
\hline
\end{tabular}

(b)

\begin{tabular}{|l|l|l|}
\hline 4 & 8 & 4 \\
\hline 4 & 4 & 4 \\
\hline 4 & 4 & 4 \\
\hline
\end{tabular}

(c)

Figure 2: a) Pixel position map in 3X3 block b) Noise pixel class map (cluster index)

\begin{tabular}{|l|l|l|}
\hline 95 & $\mathbf{2 5 1}$ & 95 \\
\hline 95 & 95 & 95 \\
\hline 95 & 95 & 95 \\
\hline
\end{tabular}

Figure 3: Pixel-wise intensity map

The pixel value for 8-bit image lies within -255 and noise pixel especially can have anyvalue fro this range in case of non linear noise present in image. For the sake of better view, non-linear salt and pepper noise compared to linear salt and pepper noise can be considered.

\subsection{Fitness function for PSO:}

$$
\begin{gathered}
\mathrm{vp}(\mathrm{i}+1)=\mathrm{h}(\mathrm{i}) * \operatorname{vp}(\mathrm{i})+\psi_{\mathrm{p}} * \operatorname{rp} *(\operatorname{xpbp}(\mathrm{i})-\mathrm{xp}(\mathrm{i}))+\psi_{\mathrm{g}} * \\
\operatorname{rg} *((\operatorname{xgbp}(\mathrm{i})-\operatorname{xp}(\mathrm{i})) \\
\mathrm{xp}(\mathrm{i}+1)=\mathrm{xp}(\mathrm{i})+\mathrm{vp}(\mathrm{i}+1)
\end{gathered}
$$

where, positive learning factors are denoted by $\psi \mathrm{p}$ and $\psi \mathrm{g}$ Random numbers in the range 0 and 1 are reepresented by rp and rg. The generation is identified by using number $\mathrm{i}$, which lies within range 1 and $I_{\text {MAX }}$ that is maximum possible generations. The inertia factor is represented by h(i). The best solution is represented by $\mathrm{fpB}(\mathrm{i})$ and $\mathrm{fgB}(\mathrm{i})$. Also, local and global best solutions are represented by $\mathrm{x}_{\mathrm{p}} \mathrm{B}(\mathrm{i})$ and $\mathrm{x}_{\mathrm{g}} \mathrm{B}(\mathrm{i})$ for $\mathrm{p}^{\text {th }}$ location and $\mathrm{i}^{\text {th }}$ generation.

3.3 Particle swarm optimization based BM3D for noise removal:

Commotion expulsion from the picture is finished by utilizing molecule swarm enhancement based streamlined BM3D strategy. The calculation of separating is as given underneath:

1. Take information shading picture

2. Separate information shading picture into blue, green and red channels

3. Get grayscale image from color input

4. Take grayscale picture for handling and noise detection

5. Classify the pixels using clustering approach

6. Perform 3x3 windowing and identify the pixels as noise pixels.

7. Identified noise windows should be compared with blue, green and red channel informations.

8. Remnove noise from each $3 \times 3$ window using optimized BM3D method.

FPGA Implementation

Table II: FPGA System configuration:

\begin{tabular}{|l|l|}
\hline Processor & Artix7 100T \\
\hline Clock frequency & $400 \mathrm{Mhz}$ \\
\hline Memory & $128 \mathrm{MB}$ \\
\hline
\end{tabular}

Published By:

Blue Eyes Intelligence Engineering

\& Sciences Publication

(C) Copyriaht: All riahts reserved. 


\section{\begin{tabular}{|l|l|}
\hline Interface type & Serial Comm \\
\hline
\end{tabular}}

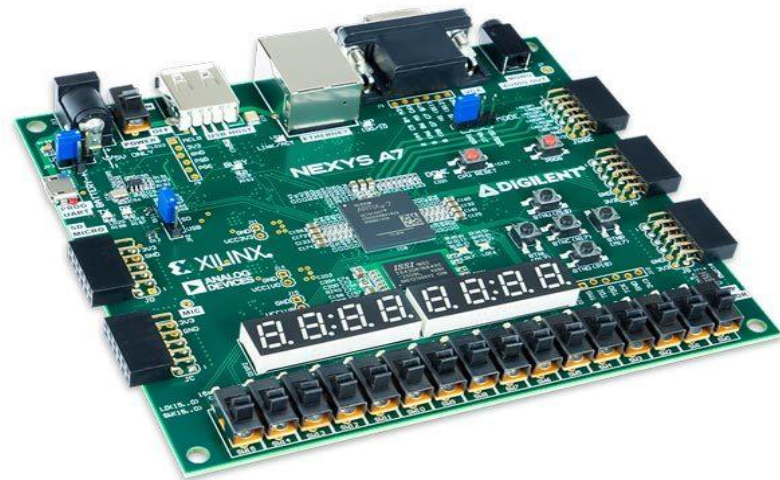

Figure 4:Artix 7 100T FPGA Experimentation board

\section{a. Soft Core processor}

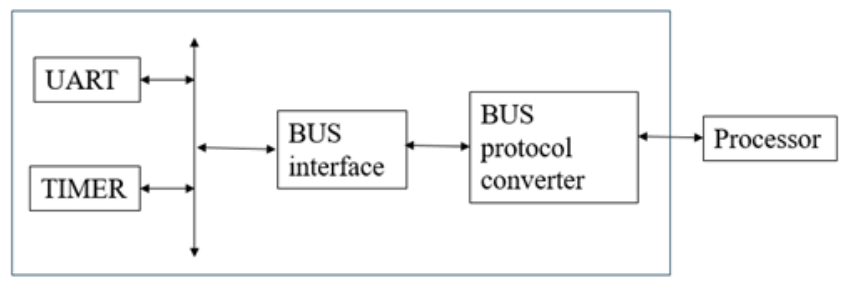

Figure 5: Soft core processor block diagram

The delicate center processor outline is appeared in figure 5 . The entire framework includes two fringe modules, transport engineering for interface, transport protocol for interfacing the processor. The UART and TIMER are used to configure the softcore processor using IP designing method and reconfigurable micro structures. When configuration is done actual UART and TIMER IP blocks are used to establish the interface. The BUS protocol has capability to change the configurations for interface between processor and IP blocks.

\section{b. Implementation Steps:}

Artix7 series Xilinx processor XC7A100T is used to implenment the proposed work. The nexus 4 DDR board consist of this required processor. The memory and UART IP interface is used in design of Microblaze softcore processor. The clock from system is used and configured to have interface with UART and memory block. The framework clock is designed according to necessity of UART, memory and Microblaze nlocks. The designed microblaze softcore processor is shown in figure 6 .

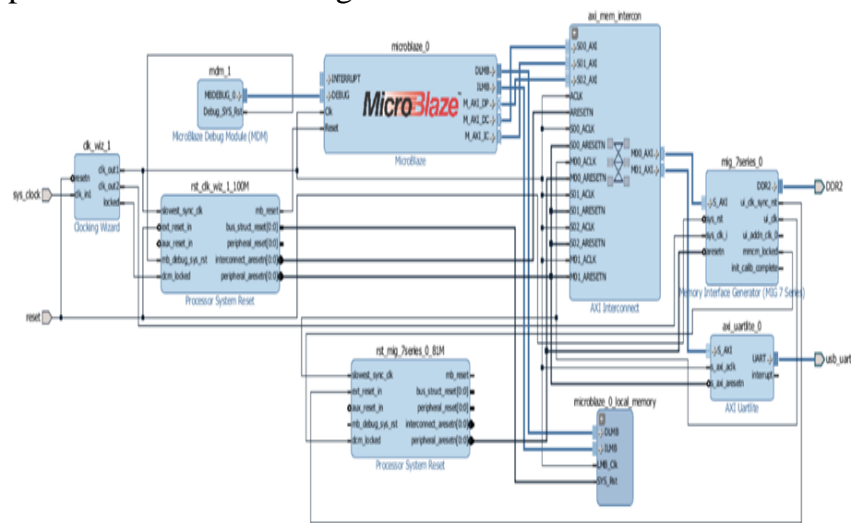

Figure 6: Softcore processor using Microblaze IP configuration

The processor design binary is obtained using Vivado design suit. The application on top of it is implemented using software development kit in vivado design and proposed processing work. The $\mathrm{C}$ programming based implementation is done. The KNN based oise pixels detection and PSO BM3D based noise removal is tested using implemented appication and results of which are taken into matlab using UART serial communication. The result sof proposed work are validated using FPGA implementation.

\section{FPGA Processing algorithm:}

1. Split red, green and blue image

2. Send each color input image serialy to FPGA board using baud rate 115200 .

3. Recieve image serialy on board.

4. Perform classification of pixels to identify the noise pixels.

5. Process classified windows with noise pixels using BM3D to remove noise.

6. Recompose image to form single matrix

7. Send respective color matrix serialy to PC.

8. Receive image on PC for each color and recompose the color image.

9. Perform resolution enhancement

10. Perform retinex to boost the dynamicity

11. Estimate PSNR of received image

\section{RESUlTs}

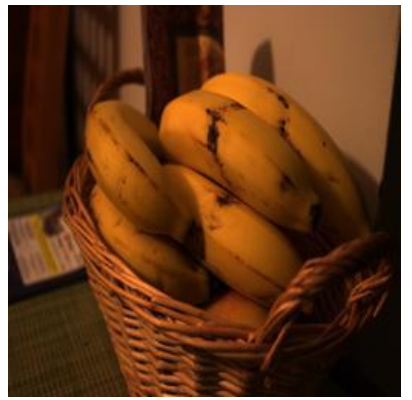

Figure 7:Input Image

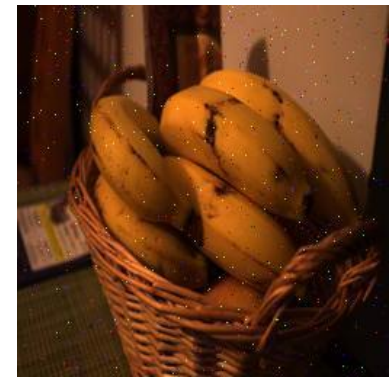

Figure 8:Noise added Image

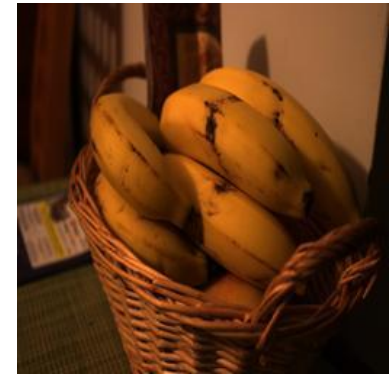

Figure 9:Optimized BM3D output (noise removed)

Published By:

Blue Eyes Intelligence Engineering \& Sciences Publication

(c) Copyright: All rights reserved.

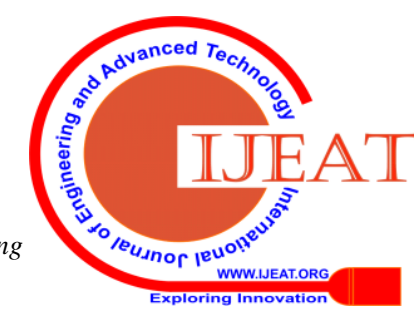




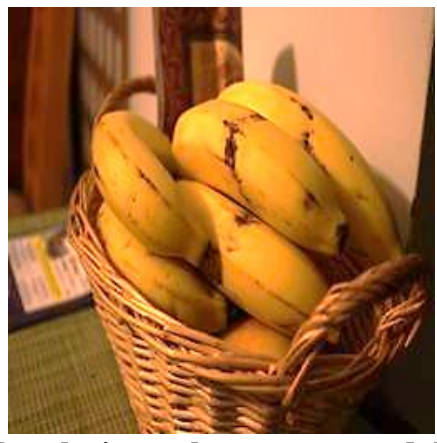

Figure 10:Resolution enhancement and debluring of butterfly image using optimized BM3D

Retinex is utilized For represnting picture with dynamic brightening. In PC vision shading consistency is an alluring element. A few retinex calculations are accessible for dynamic illunmination. We have utilized multiscale retinex for dynamic light of goals ehahnced picture which likewise shows huge contrast in PSNR values.

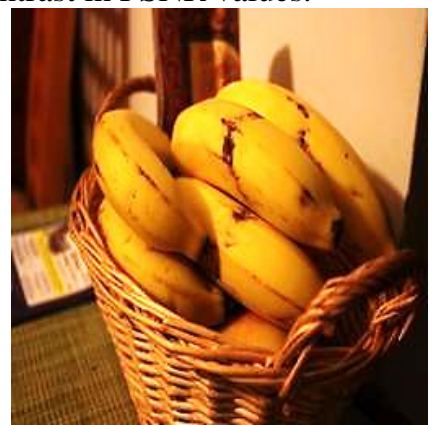

Figure 11:Output of retinex stage

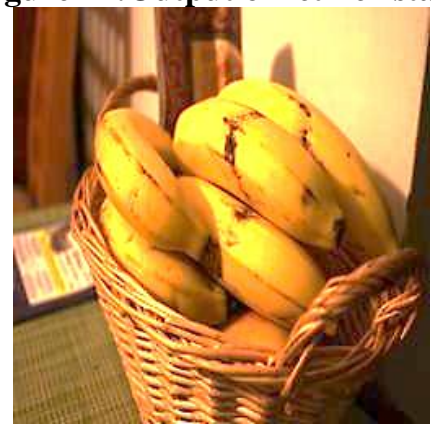

Figure 12: Output of FPGA processing

The results are evaluated using peak signal to noise ratio (PSNR) paramter along with mean square error (MSE) analysis.Th results are as shown in table II which shows performance of proposed method and respective comparative study.

TABLE III. PSNR ANALYSIS OF IMAGES USING IMPROVED STRATEGY

\begin{tabular}{|c|c|c|c|c|c|}
\hline Image & BM3D & $\begin{array}{c}\text { KNN + } \\
\text { Optimiz } \\
\text { ed } \\
\text { BM3D+ } \\
\text { Enhanc } \\
\text { ement }\end{array}$ & $\begin{array}{c}\text { KNN + } \\
\text { Optimized } \\
\text { BM3D + } \\
\text { Enhancemen } \\
\text { t+ Retinex }\end{array}$ & $\begin{array}{c}\text { FPGA } \\
\text { Impleme } \\
\text { ntation }\end{array}$ & Size \\
\hline Satellite & $23.45 \mathrm{~dB}$ & $36.25 \mathrm{~dB}$ & $47.68 \mathrm{~dB}$ & $43.62 \mathrm{~dB}$ & $256 \times 256$ \\
\hline Banana & $23.28 \mathrm{~dB}$ & $33.25 \mathrm{~dB}$ & $41.25 \mathrm{~dB}$ & $39.20 \mathrm{~dB}$ & $256 \times 256$ \\
\hline $\begin{array}{c}\text { Butterfl } \\
\text { y }\end{array}$ & $22.49 \mathrm{~dB}$ & $34.25 \mathrm{~dB}$ & $45.66 \mathrm{~dB}$ & $43.55 \mathrm{~dB}$ & $256 \times 256$ \\
\hline $\begin{array}{c}\text { Camera } \\
\text { man }\end{array}$ & $24.14 \mathrm{~dB}$ & $34.24 \mathrm{~dB}$ & $46.23 \mathrm{~dB}$ & $43.71 \mathrm{~dB}$ & $256 \times 256$ \\
\hline \begin{tabular}{c} 
Satellite \\
\hline $\begin{array}{c}\text { Butterfl } \\
\text { y }\end{array}$
\end{tabular} & $24.21 \mathrm{~dB}$ & $34.22 \mathrm{~dB}$ & $48.78 \mathrm{~dB}$ & $43.67 \mathrm{~dB}$ & $512 \times 512$ \\
\hline $\begin{array}{c}\text { Camera } \\
\text { man }\end{array}$ & $26.13 \mathrm{~dB}$ & $35.14 \mathrm{~dB}$ & $47.11 \mathrm{~dB}$ & $43.12 \mathrm{~dB}$ & $512 \times 512$ \\
\hline
\end{tabular}

\begin{tabular}{|l|l|l|l|l|l|}
\hline Banana & $25.66 \mathrm{~dB}$ & $36.15 \mathrm{~dB}$ & $47.55 \mathrm{~dB}$ & $43.22 \mathrm{~dB}$ & $512 \mathrm{x} 512$ \\
\hline
\end{tabular}

From the examination of the outcomes it very well may be seen that the improved BM3D alongside upgrade and retinex yield outflanks as far as PSNR and MSE. The presentation assessment of the proposed technique alongside relative strategies is finished utilizing RENOIR [18]. The dataset is consist of real low light images and normal PSNR and MSE values which are used to compare with results proposed work.

TABLE IV. PSNR AND MSE COMPARATIVE ANALYSIS OF VARIOUS METHOD WITH PROPOSED METHOD

\begin{tabular}{|c|c|c|}
\hline Method & PSNR & MSE \\
\hline BM3D+enhancement+retinex [19] & $42.21 \mathrm{~dB}$ & 0.191 \\
\hline BM3D & $24.29 \mathrm{~dB}$ & 0.331 \\
\hline BM3D+enhancement [17] & $32.26 \mathrm{~dB}$ & 0.288 \\
\hline Proposed & $46.22 \mathrm{~dB}$ & 0.123 \\
\hline FPGA & $42.965 \mathrm{~dB}$ & 0.144 \\
\hline
\end{tabular}

From the result shown in table it can be seen that proposed method outperforms in terms of PSNR and MSE and also in FPGA implementation.

\begin{tabular}{|l|r|r|r|}
\hline Resource & Utilization & \multicolumn{1}{l|}{ Available } & Utilization $\%$ \\
\hline LUT & 10130 & 63400 & 15.98 \\
LUTRAM & 1367 & 19000 & 7.19 \\
FF & 10027 & 126800 & 7.91 \\
BRAM & 18 & 135 & 13.33 \\
IO & 50 & 210 & 23.81 \\
BUFG & 5 & 32 & 15.63 \\
MMCM & 2 & 6 & 33.33 \\
PLL & 1 & 6 & 16.67 \\
\hline
\end{tabular}

Figure 13: FPGA implementation utilization analysis

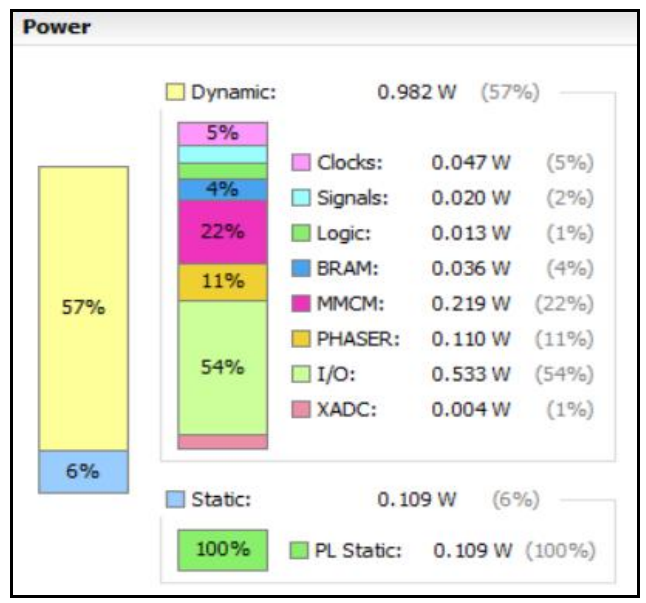

Figure 14: Power utilization report

\section{CONCLUSION}

In spite of the fact that assortment of strategies are accessible, there is necessity of advanced and viable system for nonlinear commotion expulsion. The proposed strategy right now as far as nonlinear commotion discovery and expulsion followed by the picture upgrade procedure contrasted with different strategies.

Published By:

Blue Eyes Intelligence Engineering

\& Sciences Publication

(C) Copyriaht: All riahts reserved.

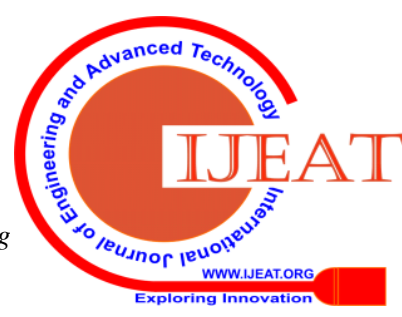


The yield results are investigated utilizing PSNR and MSE parameters and show better execution. The FPGA implementation also shows the better feasibility to implement dedicated the noise reduction and mage enhancement hardware. The results of FPGA are also satisfactory.

\section{REFERENCES}

1. D. R. K. Brownrigg, The Weighted Median Filter, Commun. ACM 27(8), 807, 1984.

2. O. Yli-Harja, J. Astola, Y. Neuvo, "Analysis of the properties of median and weighted median filters using threshold

3. logic and stack filter representation", IEEE T. Signal Process. 39(2), 395, 1991.

4. S. J. KO, Y. H Lee, Center Weighted Median Filters and Their Applications to Image Enhancement, IEEE T. Circuit. Syst. 38(9), 984, 2001.

5. T. Chen, H. R. Wu, Adaptive Impulse Detection Using Center Weighted Median Filters, IEEE Signal Precess. Lett. 8(1), 1, 2001.

6. V. Crnojevic, V. Senk, Z. Trpovski, Advanced impulse Detection based on Pixel- Wise MAD, IEEE Signal Process. Lett. 11(7), 589, 2004.

7. T. Chen, K. K. Ma, L. H. Chen, Tri- state median filter for image de noising, IEEE T. Image Process. 8(12), 1834, 1999.

8. Xiaoyin $\mathrm{Xu}$, Eric L. Miller, Dong bin Chen and Mansoor Sarhadi, “ Adaptive Two-Pass Rank Order Filter to removeImpulse Noise in Highly Corrupted Images", IEEE Trans Image Processing,Vol.13,No.2, PP.238-247, February 2004.

9. A. Buades, B. Coll, and J. M. Morel, .A review of image denoising algorithms, with a new one,. Multiscale Modeling and Simulation, vol. 4, no. 2, pp. 490.530, 2005.

10. P. Chatterjee and P. Milanfar, .Clustering-based denoising with locally learned dictionaries,. IEEE Trans. Image Process., 2008, accepted for publication.

11. K. Dabov, A. Foi, V. Katkovnik, and K. Egiazarian, .Image denoising by sparse 3D transform-domain collaborative Pltering,. IEEE Trans. Image Process., vol. 16, no. 8, pp. 2080.2095, August 2007.

12. A nonlocal and shape-adaptive transform-domain collaborative pltering,. in Proc. Local and Nonlocal Approx. in Image Process., Lausanne, Switzerland, September 2008.

13. Spatially adaptive support as a leading model-selection tool for image pltering,. in Proc. WITMSE, Tampere, Finland, August 2008.

14. M. Elad and M. Aharon, "Image denoising via sparse and redundant representations over learned dictionaries", IEEE Trans. on Image Process., vol. 15, no. 12, pp. 3736.3745, December 2006.

15. A. Foi, V. Katkovnik, and K. Egiazarian, "Pointwise Shape-Adaptive DCT for high-quality denoising and deblocking of grayscale and color images", IEEE Trans. Image Process., vol. 16, no. 5, pp. 1395.1411, May 2007.

16. Pei-Eng Ng and Kai-Kuang Ma, "A Switching Median Filter with BDND for Extremely Corrupted Images", IEEE Trans Image Processing, Vol. 15, No. 6, PP. 1506-1516, June 2006

17. Mandar D Sontakke, Meghana Kulkarni, "IMAGE NOISE REMOVAL WITH EXTENSION TO BM3D", International Journal for Control Theory and Applications, International Science Press, 2016

18. Image dataset link: https://ani.stat.fsu.edu/ abarbu/Research/T3i_Aligned.rar

19. M. D. Sontakke and M. S. Kulkarni, "Multistage combined image enhancement technique," 2016 IEEE International Conference on Recent Trends in Electronics, Information \& Communication Technology (RTEICT), Bangalore, 2016, pp. 212-216. doi: 10.1109/RTEICT.2016.7807814

\section{AUTHORS PROFILE}

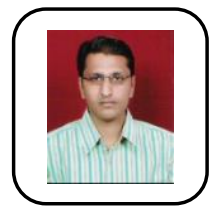

Mr. Mandar D. Sontakke, have completed his M.E. from electronics engineering in 2009 from Shivaji University. He is currently PhD scholar at department of PG studies at Vesvesvaraiya Technological University, Belgaum.

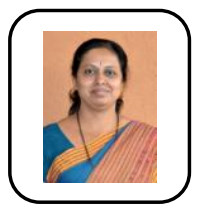

Dr. Meghana Kulkarni, Presently working as Associate Professor, in the Dept. of Electronics and Communication Engineering. VTU Belagavi. Completed Ph.D. and M.Tech. from Visvesvarya Technological University and BE from Karnataka University, Dharwad. 